UDC: $517.518 .28: 517.58$

44 (LXX) 2 (2020), 107-117

DOI: $10.37560 /$ matbil2020107a

\title{
FURTHER GENERALIZATIONS OF MINKOWSKI TYPE INEQUALITIES WITH EXTENDED MITTAG-LEFFLER FUNCTION
}

\author{
MAJA ANDRIĆ ${ }^{1}$, GHULAM FARID ${ }^{2}$, JOSIP PEČARIĆ ${ }^{3}$, \\ AND MUHAMMAD USAMA SIDDIQUE ${ }^{2}$
}

\begin{abstract}
The paper presents generalizations of Minkowski type integral inequalities for a generalized fractional integral operator containing an extended Mittag-Leffler function in the kernel.
\end{abstract}

\section{INTRODUCTION AND PRELIMINARY RESULtS}

This paper is a continuation of our work on Minkowski type inequalities, recently given in the paper [2, where we proved the reverse fractional Minkowski integral inequality using an extended Mittag-Leffler function with the corresponding fractional integral operator, as well as several related Minkowski type inequalities. The result of this paper can be applied to deduce already known fractional inequalities.

The Minkowski integral inequality is given for $f, g \in L_{p}[a, b]$ and $p \geq 1$ by

$$
\left(\int_{a}^{b}(f(x)+g(x))^{p} d x\right)^{\frac{1}{p}} \leq\left(\int_{a}^{b}(f(x))^{p} d x\right)^{\frac{1}{p}}+\left(\int_{a}^{b}(g(x))^{p} d x\right)^{\frac{1}{p}},
$$

while the Mittag-Leffler function $E_{\rho}$ is defined for $z \in \mathbb{C}$ and $\Re(\rho)>0$ by the power series using the gamma function $\Gamma$

$$
E_{\rho}(z)=\sum_{n=0}^{\infty} \frac{z^{n}}{\Gamma(\rho n+1)}
$$

Our motivation for the research on Minkowski type integral inequalities and reverse versions are the following inequalities from [3, 10]:

Theorem 1 ([3]). Let $p \geq 1$ and let $f, g \in L_{p}[a, b]$ be positive functions satisfying $0<m \leq \frac{f(x)}{g(x)} \leq M$ for $x \in[a, b]$. Then

$$
\left(\int_{a}^{b}(f(x))^{p} d x\right)^{\frac{1}{p}}+\left(\int_{a}^{b}(g(x))^{p} d x\right)^{\frac{1}{p}}
$$

2010 Mathematics Subject Classification. Primary: 26A33, 26D10, 33E12.

Key words and phrases. Minkowski inequality, Mittag-Leffler function, fractional integral operator. 


$$
\leq \frac{M(m+2)+1}{(m+1)(M+1)}\left(\int_{a}^{b}(f(x)+g(x))^{p} d x\right)^{\frac{1}{p}} .
$$

Theorem 2 ([10]). Let $p \geq 1$ and let $f, g \in L_{p}[a, b]$ be positive functions satisfying $0<m \leq \frac{f(x)}{g(x)} \leq M$ for $x \in[a, b]$. Then

$$
\begin{aligned}
& \left(\int_{a}^{b}(f(x))^{p} d x\right)^{\frac{2}{p}}+\left(\int_{a}^{b}(g(x))^{p} d x\right)^{\frac{2}{p}} \\
& \geq \frac{M(m-1)+m+1}{M}\left(\int_{a}^{b}(f(x))^{p} d x\right)^{\frac{1}{p}}\left(\int_{a}^{b}(g(x))^{p} d x\right)^{\frac{1}{p}} .
\end{aligned}
$$

The aim is to prove these kind of inequalities in the more general settings using fractional calculus, a theory of differential and integral operators of non-integer order (more on fractional calculus can be found in monographs [7, 9]). In recent years considerable interest in this theory has been stimulated by the applications that this calculus finds in numerical analysis and different areas of physics and engineering. All forms of the fractional operators (meaning fractional integral and fractional derivative) that have been studied extensively for their applications, in a special case are reduced to the left-sided and the right-sided Riemann-Liouville fractional integrals $J_{a+}^{\sigma} f$ and $J_{b-}^{\sigma} f$ of order $\sigma$ defined as in [7, 9 , for $f \in L_{1}[a, b]$ by

$$
\begin{array}{ll}
J_{a+}^{\sigma} f(x)=\frac{1}{\Gamma(\sigma)} \int_{a}^{x}(x-t)^{\sigma-1} f(t) d t, & x \in(a, b], \\
J_{b-}^{\sigma} f(x)=\frac{1}{\Gamma(\sigma)} \int_{x}^{b}(t-x)^{\sigma-1} f(t) d t, & x \in[a, b) .
\end{array}
$$

Since the Mittag-Leffler function and its generalizations appear as a solution of fractional order differential or integral equations, in [1] we presented more extended and generalized version $E_{\rho, \sigma, \tau}^{\delta, c, v}(z ; u)$ as follows:

Definition 1.1. Let $\rho, \sigma, \tau, \delta, c \in \mathbb{C}, \Re(\rho), \Re(\sigma), \Re(\tau)>0, \Re(c)>\Re(\delta)>0$ with $u \geq 0, r>0$ and $0<v \leq r+\Re(\rho)$. Then $E_{\rho, \sigma, \tau}^{\delta, c, v, r}(z ; u)$ is defined by

$$
E_{\rho, \sigma, \tau}^{\delta, c, v, r}(z ; u)=\sum_{n=0}^{\infty} \frac{\mathrm{B}_{u}(\delta+n v, c-\delta)}{\mathrm{B}(\delta, c-\delta)} \frac{(c)_{n v}}{\Gamma(\rho n+\sigma)} \frac{z^{n}}{(\tau)_{n r}} .
$$

Recall that $(c)_{n v}$ denotes the generalized Pochhammer symbol $(c)_{n v}=\frac{\Gamma(c+n v)}{\Gamma(c)}$ and $\mathrm{B}_{u}$ is an extension of the beta function

$$
\mathrm{B}_{u}(x, y)=\int_{0}^{1} t^{x-1}(1-t)^{y-1} e^{-\frac{u}{t(1-t)}} d t \quad(\Re(x), \Re(y), \Re(u)>0) .
$$

As proved in [1, the series 1.5 is absolutely convergent for all values of $z$ provided that $v<r+\Re(\rho)$. Moreover, if $v=r+\Re(\rho)$, then $E_{\rho, \sigma, \tau}^{\delta, c, v, r}(z ; u)$ converges for $|z|<\frac{r^{r} \Re(\rho)^{\Re(\rho)}}{v^{v}}$. 
Next is a corresponding generalized fractional integral operator (also defined in [1]):

Definition 1.2. Let $w, \rho, \sigma, \tau, \delta, c \in \mathbb{C}, \Re(\rho), \Re(\sigma), \Re(\tau)>0, \Re(c)>\Re(\delta)>0$ with $u \geq 0, r>0$ and $0<v \leq r+\Re(\rho)$. Let $f \in L_{1}[a, b]$ and $x \in[a, b]$. Then the generalized fractional integral operator $\varepsilon_{a^{+}, \rho, \sigma, \tau}^{w, \delta, c, v, r} f$ is defined by

$$
\left(\varepsilon_{a^{+}, \rho, \sigma, \tau}^{w, \delta, c, r} f\right)(x ; u)=\int_{a}^{x}(x-t)^{\sigma-1} E_{\rho, \sigma, \tau}^{\delta, c, r}\left(w(x-t)^{\rho} ; u\right) f(t) d t .
$$

Setting $u=0$ and $\sigma=\tau=r=q=\delta=1$ in Definition 1.1 1.5 reduces to the Mittag-Leffler function (1.2), and setting $u=\omega=0$ in Definition 1.2, (1.6) reduces to the Riemann-Liouville fractional integral $J_{a^{+}}^{\sigma} f$ of order $\sigma$, as defined in 1.3. For more details on this and how to deduce known generalizations of Mittag-Leffler function and its fractional integral operator see [1].

Further generalization of the fractional integral operator we give in [5] as follows:

Definition 1.3. Let $w, \sigma, \tau, \delta, c \in \mathbb{C}, \Re(\sigma), \Re(\tau)>0, \Re(c)>\Re(\delta)>0$ with $u \geq 0$, $\rho, r>0$ and $0<v \leq r+\rho$. Let $f \in L_{1}[a, b], 0<a<b<\infty$, be a positive function. Let $h:[a, b] \rightarrow \mathbb{R}$ be a differentiable function, strictly increasing. Also let $\frac{\phi}{x}$ be an increasing function on $[a, \infty)$ and $x \in[a, b]$. Then the generalized fractional integral operator ${ }_{h}^{\phi} F_{a^{+}, \rho, \sigma, \tau}^{w, \delta, v, r} f$ is defined by

$$
\begin{aligned}
& \left({ }_{h}^{\phi} F_{a^{+}, \rho, \sigma, \tau}^{w, \delta, c, v, r} f\right)(x ; u) \\
& \quad=\int_{a}^{x} \frac{\phi(h(x)-h(t))}{h(x)-h(t)} E_{\rho, \sigma, \tau}^{\delta, c, v, r}\left(w(h(x)-h(t))^{\rho} ; u\right) h^{\prime}(t) f(t) d t .
\end{aligned}
$$

In this paper, we will deal with a special case of the above operator, setting $\phi(x)=x^{\sigma}, \sigma>0$, in real domain. This operator we denote ${ }_{h} \Upsilon_{a^{+}, \rho, \sigma, \tau}^{w, \delta, r} f$ and define as follows.

Definition 1.4. Let $w \in \mathbb{R}, \rho, \sigma, \tau, r>0, c>\delta>0$ with $u \geq 0$ and $0<v \leq r+\rho$. Let $f \in L_{1}[a, b]$ be a positive function and $h:[a, b] \rightarrow \mathbb{R}$ be a differentiable function, strictly increasing. Then for $x \in[a, b]$ the generalized fractional integral operator ${ }_{h} \Upsilon_{a^{+}, \rho, \sigma, \tau}^{w, \delta, c, v} f$ is defined by

$$
\begin{aligned}
& \left({ }_{h} \Upsilon_{a^{+}, \rho, \sigma, \tau}^{w, \delta, c, v, r} f\right)(x ; u) \\
& \quad=\int_{a}^{x}(h(x)-h(t))^{\sigma-1} E_{\rho, \sigma, \tau}^{\delta, c, v, r}\left(w(h(x)-h(t))^{\rho} ; u\right) h^{\prime}(t) f(t) d t .
\end{aligned}
$$

If we set $u=\omega=0$ in this definition, then (1.7) reduces to the left-sided Riemann-Liouville fractional integral of a function $f$ with respect to another function $h$ of order $\sigma([7,9])$ :

$$
J_{a+; h}^{\sigma} f(x)=\frac{1}{\Gamma(\sigma)} \int_{a}^{x}(h(x)-h(t))^{\sigma-1} h^{\prime}(t) f(t) d t, \quad x \in(a, b] .
$$


The paper is organized in the following way: after this introductory section with preliminary results, further generalizations of reverse Minkowski type integral inequalities for a generalized fractional integral operator ${ }_{h} \Upsilon_{a^{+}, \rho, \sigma, \tau}^{w, \delta, c, r} f$ containing an extended Mittag-Leffler function $E_{\rho, \sigma, \tau}^{\delta, c, v, r}$ in the kernel are given in Section 2. In Section 3 we prove some related fractional Minkowski type integral inequalities. More similar results on the Minkowski type inequalities using fractional operators can be seen in 2 , 4, 6, 8, 11, 12, 13.

For the reader's convenience we will use a simplified notation

$$
\begin{aligned}
\boldsymbol{E}(z ; u) & :=E_{\rho, \sigma, \tau}^{\delta, c, v, r}(z ; u), \\
\left({ }_{\boldsymbol{h}} \Upsilon f\right)(x ; u) & :=\left({ }_{h} \Upsilon_{a^{+}, \rho, \sigma, \tau}^{w, \delta, r} f\right)(x ; u) .
\end{aligned}
$$

\section{Further generalization of Reverse Minkowski type inequalities} INVOLVING EXTENDED MitTAG-LEFFleR FUnCTION

To generalize Theorem 1 using fractional calculus, we supplement methods from the paper by L. Bougoffa ([3]) with the necessary steps.

Theorem 3. Let $w \in \mathbb{R}, \rho, \sigma, \tau, r>0, c>\delta>0$ with $u \geq 0$ and $0<v \leq$ $r+\rho$. Let $h:[a, b] \rightarrow \mathbb{R}$ be a differentiable, strictly increasing function and let $f, g, \varphi_{1}, \varphi_{2} \in L_{p}[a, b]$ be positive functions satisfying

$$
0<\varphi_{1}(x) \leq \frac{f(x)}{g(x)} \leq \varphi_{2}(x), \quad x \in[a, b]
$$

Then for $p \geq 1$ the following inequality holds

$$
\begin{aligned}
& \left.\left.\left[{ }_{\boldsymbol{h}} \Upsilon \boldsymbol{} f^{p}\right)(x ; u)\right]^{\frac{1}{p}}+\left[{ }_{\boldsymbol{h}} \Upsilon g^{p}\right)(x ; u)\right]^{\frac{1}{p}} \\
& \leq \quad\left[\left({ }_{\boldsymbol{h}} \Upsilon\left(\frac{\varphi_{2}}{1+\varphi_{2}}\right)^{p}(f+g)^{p}\right)(x ; u)\right]^{\frac{1}{p}} \\
& \quad+\left[\left({ }_{\boldsymbol{h}} \Upsilon\left(\frac{1}{1+\varphi_{1}}\right)^{p}(f+g)^{p}\right)(x ; u)\right]^{\frac{1}{p}} .
\end{aligned}
$$

Proof. Let $t \in[a, b]$. From $\frac{f(t)}{g(t)} \leq \varphi_{2}(t)$ we have

$$
f(t) \leq \varphi_{2}(t)[f(t)+g(t)]-\varphi_{2}(t) f(t),
$$

and for $p \geq 1$

$$
(f(t))^{p} \leq\left(\frac{\varphi_{2}(t)}{1+\varphi_{2}(t)}\right)^{p}[f(t)+g(t)]^{p} .
$$

Multiplying both sides of the above inequality by

$$
(h(x)-h(t))^{\sigma-1} \boldsymbol{E}\left(w(h(x)-h(t))^{\rho} ; u\right) h^{\prime}(t)
$$

and integrating on $[a, x]$ with respect to the variable $t$, we obtain

$$
\left.{ }_{(\boldsymbol{h}} \Upsilon f^{p}\right)(x ; u) \leq\left({ }_{\boldsymbol{h}} \Upsilon\left(\left(\frac{\varphi_{2}}{1+\varphi_{2}}\right)^{p}(f+g)^{p}\right)\right)(x ; u) .
$$


from which we get

$$
\left[\left({ }_{\boldsymbol{h}} \Upsilon f^{p}\right)(x ; u)\right]^{\frac{1}{p}} \leq\left[\left({ }_{\boldsymbol{h}} \Upsilon\left(\left(\frac{\varphi_{2}}{1+\varphi_{2}}\right)^{p}(f+g)^{p}\right)\right)(x ; u)\right]^{\frac{1}{p}} .
$$

Further, from $\frac{f(t)}{g(t)} \geq \varphi_{1}(t)$ follows

$$
g(t) \leq \frac{1}{\varphi_{1}(t)}[f(t)+g(t)]-\frac{1}{\varphi_{1}(t)} g(t),
$$

and if $p \geq 1$, then

$$
(g(t))^{p} \leq\left(\frac{1}{1+\varphi_{1}(t)}\right)^{p}[f(t)+g(t)]^{p} .
$$

Similarly, if we multiply above inequality by (2.3) and integrate on $[a, x]$, then we get

and also

$$
\left({ }_{\boldsymbol{h}} \Upsilon g^{p}\right)(x ; u) \leq\left({ }_{\boldsymbol{h}} \Upsilon\left(\left(\frac{1}{1+\varphi_{1}}\right)^{p}(f+g)^{p}\right)\right)(x ; u)
$$

$$
\left[\left({ }_{\boldsymbol{h}} \Upsilon g^{p}\right)(x ; u)\right]^{\frac{1}{p}} \leq\left[\left({ }_{\boldsymbol{h}} \Upsilon\left(\left(\frac{1}{1+\varphi_{1}}\right)^{p}(f+g)^{p}\right)\right)(x ; u)\right]^{\frac{1}{p}} .
$$

By adding (2.4) and (2.6), the resulting inequality (2.1) follows.

Setting $\varphi_{1}$ and $\varphi_{2}$ to be constant functions, i.e. $\varphi_{1}(x)=m$ and $\varphi_{2}(x)=M$ for all $x \in[a, b]$, we obtain the following result.

Corollary 3.1. Let $w \in \mathbb{R}, \rho, \sigma, \tau, r>0, c>\delta>0$ with $u \geq 0$ and $0<v \leq$ $r+\rho$. Let $h:[a, b] \rightarrow \mathbb{R}$ be a differentiable, strictly increasing function and let $f, g \in L_{p}[a, b]$ be positive functions satisfying

$$
0<m \leq \frac{f(x)}{g(x)} \leq M, \quad x \in[a, b] .
$$

Then for $p \geq 1$ the following inequality holds

$$
\begin{aligned}
& {\left[\left({ }_{\boldsymbol{h}} \Upsilon f^{p}\right)(x ; u)\right]^{\frac{1}{p}}+\left[\left({ }_{\boldsymbol{h}} \Upsilon g^{p}\right)(x ; u)\right]^{\frac{1}{p}}} \\
& \leq c_{1}\left[\left({ }_{\boldsymbol{h}} \Upsilon(f+g)^{p}\right)(x ; u)\right]^{\frac{1}{p}},
\end{aligned}
$$

where

$$
c_{1}=\frac{M(m+2)+1}{(m+1)(M+1)} .
$$

Remark 2.1: If the function $h$ is the identity function, then we obtain an inequality for the generalized fractional operator $\varepsilon_{a^{+}, \rho, \sigma, \tau}^{w, \delta, c, v} f$ from Definition 1.2 .

$$
\begin{aligned}
& {\left[\left(\varepsilon_{a^{+}, \rho, \sigma, \tau}^{w, \delta, c, v, r} f^{p}\right)(x ; u)\right]^{\frac{1}{p}}+\left[\left(\varepsilon_{a^{+}, \rho, \sigma, \tau}^{w, \delta, c, v, r} g^{p}\right)(x ; u)\right]^{\frac{1}{p}}} \\
& \quad \leq c_{1}\left[\left(\varepsilon_{a^{+}, \rho, \sigma, \tau}^{w, \delta, c, v, r}(f+g)^{p}\right)(x ; u)\right]^{\frac{1}{p}}
\end{aligned}
$$


where $c_{1}$ is defined by 2.8 .

This inequality is given in [2, Theorem 2.1].

Next theorem is a fractional generalization of Theorem 2, It follows by the use of the Minkowski integral inequality (1.1).

Theorem 4. Suppose the assumptions of Theorem 3 hold. Then

$$
\begin{aligned}
& \left.\left[\left({ }_{\boldsymbol{h}} \Upsilon f^{p}\right)(x ; u)\right]^{\frac{2}{p}}+\left[{ }_{\boldsymbol{h}} \Upsilon \boldsymbol{g} g^{p}\right)(x ; u)\right]^{\frac{2}{p}} \\
& \left.\geq\left[\left({ }_{\boldsymbol{h}} \Upsilon\left(\frac{1+\varphi_{2}}{\varphi_{2}}\right)^{p} f^{p}\right)(x ; u)\right]^{\frac{1}{p}}\left[{ }_{\boldsymbol{h}} \Upsilon\left(1+\varphi_{1}\right)^{p} g^{p}\right)(x ; u)\right]^{\frac{1}{p}} \\
& \left.-2\left[\left({ }_{\boldsymbol{h}} \Upsilon f^{p}\right)(x ; u)\right]^{\frac{1}{p}}\left[{ }_{(\boldsymbol{h}} \Upsilon g^{p}\right)(x ; u)\right]^{\frac{1}{p}} .
\end{aligned}
$$

Proof. For $p \geq 1$ and $t \in[a, b]$, inequalities 2.2 and 2.5 can also be written as

$$
\left(\frac{1+\varphi_{2}(t)}{\varphi_{2}(t)}\right)^{p}\left((f(t))^{p} \leq[f(t)+g(t)]^{p}\right.
$$

and

$$
\left(1+\varphi_{1}(t)\right)^{p}(g(t))^{p} \leq[f(t)+g(t)]^{p} .
$$

If we multiply both sides of each inequality by $(2.3)$, integrate on $[a, x]$ with respect to the variable $t$ and use power to the $\frac{1}{p}$, then we obtain

$$
\left.\left[\left({ }_{\boldsymbol{h}} \Upsilon\left(\left(\frac{1+\varphi_{2}}{\varphi_{2}}\right)^{p} f^{p}\right)\right)(x ; u)\right]^{\frac{1}{p}} \leq\left[{ }_{\boldsymbol{h}} \Upsilon(f+g)^{p}\right)(x ; u)\right]^{\frac{1}{p}}
$$

and

$$
\left[\left({ }_{\boldsymbol{h}} \Upsilon\left(\left(1+\varphi_{1}\right)^{p} g^{p}\right)\right)(x ; u)\right]^{\frac{1}{p}} \leq\left[\left({ }_{\boldsymbol{h}} \Upsilon(f+g)^{p}\right)(x ; u)\right]^{\frac{1}{p}}
$$

Taking the product of the inequalities 2.10 and 2.11 we obtain

$$
\begin{aligned}
& {\left[\left({ }_{\boldsymbol{h}} \Upsilon\left(\left(\frac{1+\varphi_{2}}{\varphi_{2}}\right)^{p} f^{p}\right)\right)(x ; u)\right]^{\frac{1}{p}}\left[\left({ }_{\boldsymbol{h}} \Upsilon\left(\left(1+\varphi_{1}\right)^{p} g^{p}\right)\right)(x ; u)\right]^{\frac{1}{p}}} \\
& \left.\leq\left[{ }_{\boldsymbol{h}} \Upsilon(f+g)^{p}\right)(x ; u)\right]^{\frac{2}{p}} .
\end{aligned}
$$

If we apply Minkowski's inequality on right hand side, we get

$$
\begin{aligned}
& {\left[\left({ }_{\boldsymbol{h}} \Upsilon\left(\left(\frac{1+\varphi_{2}}{\varphi_{2}}\right)^{p} f^{p}\right)\right)(x ; u)\right]^{\frac{1}{p}}\left[\left({ }_{\boldsymbol{h}} \Upsilon\left(\left(1+\varphi_{1}\right)^{p} g^{p}\right)\right)(x ; u)\right]^{\frac{1}{p}}} \\
& \left.\leq\left[\left(\left({ }_{\boldsymbol{h}} \Upsilon f^{p}\right)(x ; u)\right)^{\frac{1}{p}}+\left({ }_{\boldsymbol{h}} \Upsilon \boldsymbol{g} g^{p}\right)(x ; u)\right)^{\frac{1}{p}}\right]^{2} .
\end{aligned}
$$

From this we can easily obtain the inequality 2.9 .

If $\varphi_{1}(x)=m$ and $\varphi_{2}(x)=M$ for all $x \in[a, b]$, then the next inequality follows.

Corollary 4.1. Suppose the assumptions of Corollary 3.1 hold. Then

$$
\begin{aligned}
& \left.\left[\left({ }_{\boldsymbol{h}} \Upsilon f^{p}\right)(x ; u)\right]^{\frac{2}{p}}+\left[{ }_{\boldsymbol{h}} \Upsilon \boldsymbol{g} g^{p}\right)(x ; u)\right]^{\frac{2}{p}} \\
& \left.\geq c_{2}\left[\left({ }_{\boldsymbol{h}} \Upsilon f^{p}\right)(x ; u)\right]^{\frac{1}{p}}\left[{ }_{(\boldsymbol{h}} \Upsilon g^{p}\right)(x ; u)\right]^{\frac{1}{p}},
\end{aligned}
$$


where

$$
c_{2}=\frac{M(m-1)+m+1}{M} .
$$

Remark 2.2: If the function $h$ is the identity function, then we obtain [2, Theorem 2.2], an inequality for the generalized fractional operator $\varepsilon_{a^{+}, \rho, \sigma, \tau}^{w, c, c, r} f$.

\section{Related fractional Minkowski type integral ineQualities}

We continue with the generalizations of the reverse Minkowski type integral inequalities. Starting conditions that we will need in this section are those given in Corollary 3.1 where we have $0<m \leq \frac{f(x)}{g(x)} \leq M$.

Theorem 5. Suppose that assumptions of Corollary 3.1 hold. Let $p, q>1$ with $\frac{1}{p}+\frac{1}{q}=1$. Then

$$
\left.\left.\left[{ }_{\boldsymbol{h}} \Upsilon \mathbf{f}\right)(x ; u)\right]^{\frac{1}{p}}\left[{ }_{\boldsymbol{h}} \Upsilon \mathbf{\Upsilon} g\right)(x ; u)\right]^{\frac{1}{q}} \leq\left(\frac{M}{m}\right)^{\frac{1}{p q}}\left({ }_{\boldsymbol{h}} \Upsilon\left(f^{\frac{1}{p}} g^{\frac{1}{q}}\right)\right)(x ; u) .
$$

Proof. Let $t \in[a, b]$. From $\frac{f(t)}{g(t)} \leq M$ we obtain

$$
(f(t))^{\frac{1}{q}} \leq M^{\frac{1}{q}}(g(t))^{\frac{1}{q}}
$$

and after multiplication by $(f(t))^{\frac{1}{p}}$ we get

$$
f(t) \leq M^{\frac{1}{q}}(f(t))^{\frac{1}{p}}(g(t))^{\frac{1}{q}}
$$

If we multiply both sides of the above inequality by $(2.3)$, integrate on $[a, x]$ with respect to the variable $t$ and use power to the $\frac{1}{p}$, then we obtain

$$
\left[\left({ }_{\boldsymbol{h}} \Upsilon f\right)(x ; u)\right]^{\frac{1}{p}} \leq M^{\frac{1}{p q}}\left[\left({ }_{h} \Upsilon\left(f^{\frac{1}{p}} g^{\frac{1}{q}}\right)\right)(x ; u)\right]^{\frac{1}{p}} .
$$

Next from the lower bound $m \leq \frac{f(t)}{g(t)}$ we have

$$
g(t) \leq m^{-\frac{1}{p}}(f(t))^{\frac{1}{p}}(g(t))^{\frac{1}{q}} .
$$

Again, if we multiply both sides of the above inequality by (2.3), integrate on $[a, x]$ with respect to the variable $t$ and use power to the $\frac{1}{p}$, then we get

$$
\left.\left[{ }_{\boldsymbol{h}} \Upsilon \mathrm{\Upsilon} g\right)(x ; u)\right]^{\frac{1}{q}} \leq m^{-\frac{1}{p q}}\left[\left({ }_{h} \Upsilon\left(f^{\frac{1}{p}} g^{\frac{1}{q}}\right)\right)(x ; u)\right]^{\frac{1}{q}} .
$$

The inequality (3.1) now follows from the product of inequalities $(3.2)$ and $(3.3)$.

In the next theorem we will need the following elementary inequality for $x, y \geq 0$ and $p>1$ :

$$
(x+y)^{p} \leq 2^{p-1}\left(x^{p}+y^{p}\right),
$$

along with the well known Young's inequality for $x, y \geq 0$ and $p, q>1$ such that $\frac{1}{p}+\frac{1}{q}=1$ :

$$
x y \leq \frac{x^{p}}{p}+\frac{y^{q}}{q} .
$$


Theorem 6. Suppose that assumptions of Corollary 3.1 hold. Let $p, q>1$ with $\frac{1}{p}+\frac{1}{q}=1$. Then

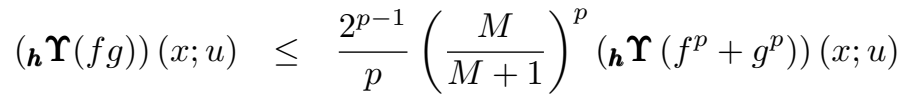

$$
\begin{aligned}
& +\frac{2^{q-1}}{q}\left(\frac{1}{m+1}\right)^{q}\left({ }_{\boldsymbol{h}} \Upsilon\left(f^{q}+g^{q}\right)\right)(x ; u) .
\end{aligned}
$$

Proof. Let $t \in[a, b], p, q>1$ and $\frac{1}{p}+\frac{1}{q}=1$. From $\frac{f(t)}{g(t)} \leq M$ we get

$$
(f(t))^{p} \leq\left(\frac{M}{M+1}\right)^{p}[f(t)+g(t)]^{p} .
$$

If we multiply both sides of the above inequality by 2.3 and integrate on $[a, x]$ with respect to the variable $t$, then we obtain

$$
\frac{1}{p}\left({ }_{\boldsymbol{h}} \Upsilon f^{p}\right)(x ; u) \leq \frac{1}{p}\left(\frac{M}{M+1}\right)^{p}\left({ }_{\boldsymbol{h}} \Upsilon \boldsymbol{\Upsilon}(f+g)^{p}\right)(x ; u)
$$

Next from $m \leq \frac{f(t)}{g(t)}$ we obtain

$$
(g(t))^{q} \leq\left(\frac{1}{m+1}\right)^{q}[f(t)+g(t)]^{q} .
$$

Again, if we multiply both sides of the above inequality by $(2.3)$ and integrate on $[a, x]$ with respect to the variable $t$, then we get

$$
\left.\frac{1}{q}{ }_{(\boldsymbol{h}} \boldsymbol{\Upsilon} g^{q}\right)(x ; u) \leq \frac{1}{q}\left(\frac{1}{m+1}\right)^{q}\left({ }_{\boldsymbol{h}} \boldsymbol{\Upsilon}(f+g)^{q}\right)(x ; u)
$$

Using Young's inequality (3.5) we have

$$
f(t) g(t) \leq \frac{(f(t))^{p}}{p}+\frac{(g(t))^{q}}{q} .
$$

Multiplying both sides of the above inequality by $(2.3)$ and integrating on $[a, x]$ we get

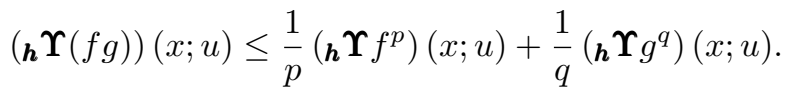

From (3.7), 3.8 and 3.9 we obtain

$$
\begin{aligned}
& \left.\left.{ }_{(\boldsymbol{h}} \Upsilon \boldsymbol{\Upsilon}(f g)\right)(x ; u) \leq \frac{1}{p}\left(\frac{M}{M+1}\right)^{p}{ }_{(\boldsymbol{h}} \Upsilon \boldsymbol{\Upsilon}(f+g)^{p}\right)(x ; u) \\
& +\frac{1}{q}\left(\frac{1}{m+1}\right)^{q}\left({ }_{\boldsymbol{h}} \Upsilon \boldsymbol{\Upsilon}(f+g)^{q}\right)(x ; u) .
\end{aligned}
$$

Using elementary inequality (3.4) we obtain

$$
\left({ }_{\boldsymbol{h}} \Upsilon(f+g)^{p}\right)(x ; u) \leq 2^{p-1}\left({ }_{\boldsymbol{h}} \Upsilon\left(f^{p}+g^{p}\right)\right)(x ; u)
$$

and

$$
\left.\left.{ }_{(\boldsymbol{h}} \Upsilon(f+g)^{q}\right)(x ; u) \leq 2^{q-1}{ }_{(\boldsymbol{h}} \Upsilon\left(f^{q}+g^{q}\right)\right)(x ; u)
$$


Hence, from (3.10), (3.11) and (3.12) we obtain (3.6).

Next theorem needs a simple application of the given condition (2.7).

Theorem 7. Suppose that assumptions of Corollary 3.1 hold. Then

$$
\begin{aligned}
\left.\frac{1}{M}{ }_{\boldsymbol{h}} \boldsymbol{\Upsilon}(f g)\right)(x ; u) & \leq \frac{1}{(m+1)(M+1)}\left({ }_{\boldsymbol{h}} \boldsymbol{\Upsilon}(f+g)^{2}\right)(x ; u) \\
& \left.\leq \frac{1}{m}{ }_{\boldsymbol{h}} \boldsymbol{\Upsilon}(f g)\right)(x ; u) .
\end{aligned}
$$

Proof. From $0<m \leq \frac{f(t)}{g(t)} \leq M$ and $\frac{1}{M} \leq \frac{g(t)}{f(t)} \leq \frac{1}{m}$ we obtain

$$
(m+1) g(t) \leq f(t)+g(t) \leq(M+1) g(t)
$$

and

$$
\left(\frac{M+1}{M}\right) f(t) \leq f(t)+g(t) \leq\left(\frac{m+1}{m}\right) f(t) .
$$

Multiplying inequalities (3.14) and (3.15) we get

$$
\frac{1}{M} f(t) g(t) \leq \frac{(f(t)+g(t))^{2}}{(M+1)(m+1)} \leq \frac{1}{m} f(t) g(t)
$$

Inequalities 3.13 now follow if we multiply the above by 2.3 and integrate on $[a, x]$ with respect to the variable $t$.

In the last theorem we add a positive parameter $\vartheta$ such that $\vartheta<m$, i.e.

$$
0<\vartheta<m \leq \frac{f(x)}{g(x)} \leq M, \quad x \in[a, b] .
$$

Theorem 8. Suppose that assumptions of Corollary 3.1 hold. Let $\vartheta>0$ be such that $\vartheta<m$ in 2.7 . Then

$$
\begin{aligned}
& \frac{M+1}{M-\vartheta}\left({ }_{\boldsymbol{h}} \Upsilon \boldsymbol{}(f-\vartheta g)^{\frac{1}{p}}\right)(x ; u) \\
& \left.\left.\quad \leq\left[{ }_{\boldsymbol{h}} \Upsilon \boldsymbol{\Upsilon} f^{p}\right)(x ; u)\right]^{\frac{1}{p}}+\left[{ }_{\boldsymbol{h}} \boldsymbol{\Upsilon} g^{p}\right)(x ; u)\right]^{\frac{1}{p}} \\
& \quad \leq \frac{m+1}{m-\vartheta}\left({ }_{\boldsymbol{h}} \Upsilon \boldsymbol{\Upsilon}(f-\vartheta g)^{\frac{1}{p}}\right)(x ; u) .
\end{aligned}
$$

Proof. From the given condition $0<\vartheta<m \leq M$, we have

$$
m-M \vartheta \leq M-m \vartheta,
$$

from which follow

and

$$
\begin{gathered}
\frac{M+1}{M-\vartheta} \leq \frac{m+1}{m-\vartheta}, \\
m-\vartheta \leq \frac{f(t)-\vartheta g(t)}{g(t)} \leq M-\vartheta
\end{gathered}
$$

$$
\frac{[f(t)-\vartheta g(t)]^{p}}{(M-\vartheta)^{p}} \leq(g(t))^{p} \leq \frac{[f(t)-\vartheta g(t)]^{p}}{(m-\vartheta)^{p}} .
$$


If we multiply above inequalities by (2.3), integrate on $[a, x]$ with respect to the variable $t$ and use power to the $\frac{1}{p}$, then we get

$$
\begin{aligned}
& \frac{1}{M-\vartheta}\left[\left({ }_{\boldsymbol{h}} \Upsilon \boldsymbol{(}(f-\vartheta g)^{p}\right)(x ; u)\right]^{\frac{1}{p}} \\
& \leq\left[\left({ }_{\boldsymbol{h}} \boldsymbol{\Upsilon} g^{p}\right)(x ; u)\right]^{\frac{1}{p}} \\
& \left.\leq \frac{1}{m-\vartheta}\left[{ }_{\boldsymbol{h}} \Upsilon \boldsymbol{\Upsilon}(f-\vartheta g)^{p}\right)(x ; u)\right]^{\frac{1}{p}} .
\end{aligned}
$$

Further, from $\frac{1}{M} \leq \frac{g(t)}{f(t)} \leq \frac{1}{m}$ we get

$$
\frac{m-\vartheta}{m} \leq \frac{f(t)-\vartheta g(t)}{f(t)} \leq \frac{M-\vartheta}{M}
$$

from which we have

$$
\frac{M^{p}[f(t)-\vartheta g(t)]^{p}}{(M-\vartheta)^{p}} \leq(f(t))^{p} \leq \frac{m^{p}[f(t)-\vartheta g(t)]^{p}}{(m-\vartheta)^{p}} .
$$

Again, multiplying above inequalities by $(2.3)$, integrating on $[a, x]$ and using power to the $\frac{1}{p}$, we obtain

$$
\begin{aligned}
& \frac{M}{M-\vartheta}\left[\left({ }_{\boldsymbol{h}} \Upsilon \boldsymbol{\Upsilon}(f-\vartheta g)^{p}\right)(x ; u)\right]^{\frac{1}{p}} \\
& \left.\leq\left[{ }_{\boldsymbol{h}} \Upsilon \boldsymbol{f} f^{p}\right)(x ; u)\right]^{\frac{1}{p}} \\
& \left.\leq \frac{m}{m-\vartheta}\left[{ }_{h} \Upsilon(f-\vartheta g)^{p}\right)(x ; u)\right]^{\frac{1}{p}} .
\end{aligned}
$$

By adding the inequalities (3.17) and (3.18), we get (3.16).

Remark 3.1: If in the obtained results of this section we use the identity function for the function $h$, then we obtain Theorem 3.1-Theorem 3.4 from [2], i.e. inequalities for the generalized fractional operator $\varepsilon_{a^{+}, \rho, \sigma, \tau}^{w, \delta, c, r} f$ from Definition 1.2 .

At the end we emphasize that the right-sided versions of all inequalities in this paper can be established using

$$
\begin{aligned}
& \left({ }_{h} \Upsilon_{b^{-}, \rho, \sigma, \tau}^{w, \delta, c, r} f\right)(x ; u) \\
& \quad=\int_{x}^{b}(h(t)-h(x))^{\sigma-1} E_{\rho, \sigma, \tau}^{\delta, c, v, r}\left(w(h(t)-h(x))^{\rho} ; u\right) h^{\prime}(t) f(t) d t .
\end{aligned}
$$

and proved analogously.

Acknowledgements. This research is partially supported through project KK.01.1.1.02.0027, a project co-financed by the Croatian Government and the European Union through the European Regional Development Fund - the Competitiveness and Cohesion Operational Programme, by the Higher Education Commission of Pakistan under NRPU 2016, Project No. 5421 and the Ministry of Education and Science of the Russian Federation (the Agreement No. 02.a03.21.0008). 


\section{REFERENCES}

[1] M. Andrić, G. Farid and J. Pečarić, A further extension of Mittag-Leffler function, Fract. Calc. Appl. Anal. 21, 4 (2018), 1377-1395.

[2] M. Andrić, G. Farid, J. Pečarić and U. Siddique, Generalized Minkowski-type fractional inequalities involving extended Mittag-Leffler function, J. Indian Math. Soc. 87, 3-4 (2020), $137-147$.

[3] L. Bougoffa, On Minkowski and Hardy integral inequalities, J. Inequal. Pure Appl. Math. 7, 2 (2006), Article 60.

[4] V. L. Chinchane, New approach of Minkowski fractional inequalities using generalized $k$ fractional integral operator, J. Indian Math. Soc. 85, 1-2 (2018), 32-41.

[5] G. Farid, A unified integral operator and further its consequences, Open J. Math. Anal. 4, 1 (2020), 1-7.

[6] H. Khan, T. Abdeljawad, C. Tunç, A. Alkhazzan and A. Khan, Minkowski's inequality for the AB-fractional integral operator, J Inequal. Appl. (2019) 2019: 96.

[7] A. A. Kilbas, H. M. Srivastava, J. J. Trujillo, Theory and Applications of Fractional Differential Equations, Elsevier, Amsterdam, Netherlands (2006).

[8] G. Rahman, A. Khan, T. Abdeljawad and K. S. Nisar, The Minkowski inequalities via generalized proportional fractional integral operators, Adv. Diff. Equ. (2019), 2019: 287.

[9] S. G. Samko, A. A. Kilbas, O. I. Marichev, Fractional Integrals and Derivatives. Theory and Applications, Gordon and Breach, Reading (1993).

[10] E. Set, M Özdemir and S. S. Dragomir, On the Hermite-Hadamard inequality and other integral inequalities involving two functions, J. Inequal. Appl. (2010), 2010: 148102.

[11] J. Vanterler da C. Sousa and E. Capelas de Oliveira, The Minkowski's inequality by means of a generalized fractional integral, AIMS Mathematics 3, 1 (2018), 131-147.

[12] B. Sroysang, More on reverses of Minkowski's integral inequality, Math. Aeterna 3, 7 (2013), $597-600$

[13] W. T. Sulaiman, Reverses of Minkowski's, Hölder's and Hardy's integral inequalities, Int. J. Modern Math. Sci 1, 1 (2012), 14-24.

1 University of Split,

Faculty of Civil Engineering, Architecture and Geodesy,

Matice hrvatske 15, 21000 Split, Croatia

Email address: maja.andric@gradst.hr

2 COMSATS University Islamabad,

Department of Mathematics,

Aтtock Campus, Pakistan

Email address: faridphdsms@hotmail.com,ghlmfarid@ciit-attock.edu.pk

Email address: usamas650@gmail.com

3 RUDN UNIVERSITY,

Miklukho-Maklaya str. 6, 117198 Moscow, Russia

Email address: pecaric@element.hr

Received 28.7.2020

Revised 13.11.2020

Accepted 15.11.2020 